\title{
A REMARK ON CURVATURE AND THE DIRICHLET PROBLEM
}

\author{
BY M. S. NARASIMHAN
}

\author{
Communicated by S. Bochner, August 17, 1959
}

1. Introduction. Let $M$ be a compact oriented Riemannian manifold with positive definite Ricci curvature. By a well-known theorem of Bochner-Myers $[2, \S 26$, p. 132] there are no nonzero harmonic forms of degree one on $M$. This result implies that "Dirichlet's problem" in the sense of $\S 6$ and $\$ 4$ of reference [1] is solvable in $M$ and that there exists the Green's form of degree one on $M$ which is an elementary kernel for the Laplacian $\Delta$ (on 1-forms) [2, §31]. We shall point out in this note how, in this form, the result can be generalized to noncompact manifolds. We use the notations of [2].

2. Theorem. Let $M$ be an oriented $C^{\infty}$ Riemannian manifold countable at infinity. We assume that the mean curvature is positive and bounded away from zero, that is we assume that there exists a constant C>0 such that

$$
R(v, v) \geqq C g(v, v)
$$

for every tangent vector $v, R(v, v)$ denoting the Ricci form and $g(v, v)$, the metric form. Then Dirichlet's problem for 1-forms is solvable on $M$ and there exists the Green's form of degree one.

Proor. Referring to Proposition IV and $\S 5$ of [1], we have only to prove the Poincaré inequality for $C^{\infty} 1$-forms with compact supports. Let $\alpha=\left(\alpha_{1}, \cdots, \alpha_{k}, \cdots, \alpha_{n}\right)$ be a $C^{\infty} 1$-form with compact support. Then

$$
(\Delta \alpha)_{k}=-\nabla^{i} \nabla_{i} \alpha_{k}-R_{k}^{h} \alpha_{k} .
$$

We have (see $[2$, p. 132]),

$$
\begin{aligned}
(\alpha, \Delta \alpha)_{L^{2}} & =-\int \alpha^{k} \nabla_{i} \nabla^{i} \alpha_{k} * 1-\int R_{k}^{h} \alpha_{h} \alpha^{k} * 1 \\
& =\int \nabla_{i} \alpha^{k} \nabla^{i} \alpha_{k} * 1-\int R_{h k} \alpha^{h} \alpha^{k} * 1,
\end{aligned}
$$

using integration by parts. By assumption we have

$$
-R_{h k} \alpha^{h} \alpha^{k} \geqq C g_{h k} \alpha^{h} \alpha^{k}
$$

and we have 


$$
\int \nabla_{i} \alpha^{k} \nabla^{i} \alpha_{k} * 1 \geqq 0
$$

Consequently

$$
(\alpha, \Delta \alpha)_{L^{2}} \geqq C \int g_{h k} \alpha^{h} \alpha^{k} * 1
$$

that is,

$$
(d \alpha, d \alpha)_{L^{2}}+(\partial \alpha, \partial \alpha)_{L^{2}} \geqq C(\alpha, \alpha)_{L^{2}}
$$

which is Poincaré's inequality for 1-forms with compact supports

\section{REFERENCES}

1. M. S. Narasimhan, The problem of limits on a Riemannian manifold, J. Indian Math. Soc. vol. 20 (1956) pp. 291-297.

2. G. de Rham, Variêtés différentiables, Paris, Hermann and Cie, 1955.

Tata Institute of Fundamental Research, Bombay, and

Centre National de la Recherche Scientifique, Paris 